\title{
Entdecken und Rechtfertigen in den Digital Humanities
}

Zusammenfassung: In diesem Beitrag werde ich die Unterscheidung zwischen Entdeckungs- und Rechtfertigungszusammenhang diskutieren und für die Methodenreflexion in den digitalen Geisteswissenschaften fruchtbar machen. Ziel ist es, eine adäquate Begrifflichkeit zu entwickeln, mit der die komplexen interdisziplinären und kollaborativen Forschungsprozesse analysiert werden können.

Abstract: In this contribution I will discuss the distinction between context of discovery and context of justification in order to make them fruitful for methodological reflection in the digital humanities. The aim is to develop adequate terminology, with which the complex interdisciplinary and collaborative research processes can be analysed.

\section{Einleitung}

Dieser Beitrag beschäftigt sich aus wissenschaftstheoretischer Perspektive mit den Elementen des Forschungsprozesses in den Digital Humanities (DH) am Beispiel der im Center for Reflected Text Analytics (CRETA) entwickelten reflektierten algorithmischen Textanalyse. Das Ziel ist es, einen begrifflichen Rahmen zu erarbeiten, um die fächerübergreifende Abstimmung der Arbeitsschritte zu erleichtern. Dies soll im Rückgriff auf den wissenschaftstheoretischen Diskurs über Entdecken und Rechtfertigen in den Wissenschaften geschehen. Dabei werden die Begriffe so weit ausdifferenziert, dass die von Pichler und Reiter (2020), in diesem Band ab Seite 43, ausgearbeiteten Arbeitsschritte funktional charakterisiert werden können. Die schematische Einordnung der infrage stehenden Arbeitsschritte kann in der Folge die interdisziplinäre Verständigung erleichtern, da es möglich wird, die jeweiligen Module hinsichtlich ihrer Leistungen und Funktionen $\mathrm{zu}$ thematisieren, ohne das Vokabular, die Methodologie und das Domänenwissen der entsprechenden Fachdisziplin replizieren zu müssen. Die funktionale Charakterisierung der Arbeitsschritte kann als Schnittstelle des interdisziplinären Austauschs dienen, wobei die Kompetenzen zur Bewertung der Schritte in den jeweiligen Fachdisziplinen verbleiben können. Somit wird eine genuin arbeitsteilige Vorgehensweise erleichtert, bei der sich nicht alle Beteilig-

Dominik Gerstorfer, Institut für Philosophie, Universität Stuttgart

つ Open Access. ( 2020 Dominik Gerstorfer, publiziert von De Gruyter (c))BY-NC-ND Dieses Werk ist lizenziert unter der Creative Commons Attribution-NonCommercial-NoDerivatives 4.0 International Lizenz. https://doi.org/10.1515/9783110693973-005 
ten das gesamte Expertenwissen der anderen Fachdisziplinen aneignen müssen, sondern sich kompetent über die Schritte zur Erreichung des gemeinsamen Forschungsziels austauschen können.

Eine solche Anwendung wissenschaftstheoretischer Konzepte auf eine konkrete Forschungspraxis ist mit zwei Schwierigkeiten konfrontiert: Zum einen beschäftigt sich die Wissenschaftstheorie primär mit der abgeschlossenen Forschung, d.h. sie analysiert wissenschaftlichen Prozesse und Ergebnisse erst im Nachhinein und trägt nur indirekt dazu bei, Prozesse zu optimieren und zukünftige Forschung zu verbessern. Zum anderen ist das Abstraktionsniveau wissenschaftstheoretischer Untersuchungen, die zumeist größtmögliche Allgemeinheit anstreben, sehr hoch und kann nicht direkt auf spezifische Praktiken angewendet werden. Darum wird es nötig sein, die allgemeinen Konzepte so zu konkretisieren, dass sie im Kontext der reflektierten Textanalyse eingesetzt werden können. Der hier vorgeschlagene Begriffsrahmen nimmt eine vermittelnde Stellung zwischen den arbeitspraktischen Erwägungen des CRETA-workflows und den theoretischen Überlegungen der Wissenschaftstheorie ein. So kann eine weitere Reflexionsebene gewonnen werden, um erkenntnistheoretische Bewertungen schon bei der Planung und Durchführung von konkreten Projekten zu berücksichtigen.

In Abschnitt 2 werde ich Ziele und Elemente wissenschaftlicher Forschung im Allgemeinen skizzieren und die speziellen Eigenarten der interdisziplinären Forschung in den Digital Humanities (DH) herausarbeiten, um die Unterscheidung zwischen Entdeckung und Rechtfertigung motivieren, die ich dann in Abschnitt 3 weiter ausführe und diskutiere. In Abschnitt 4 und Abschnitt 5 werde ich die Leitunterscheidung Entdecken/Rechtfertigen weiter ausdifferenzieren, um sie dann in Abschnitt 6 auf einzelne Komponenten des Arbeitsablaufes anzuwenden.

\section{Der Forschungsprozess}

Allgemein kann der Forschungsprozess als Praxis aufgefasst werden, die das Ziel hat, gehaltvolle und wahre Aussagen über ihren Forschungsgegenstand zu machen.

Diese knappe Charakterisierung bedarf einer Erläuterung: Gehaltvoll heißen Aussagen dann, wenn aus ihnen weitere Aussagen über den Untersuchungsgegenstand abgeleitet werden können. Damit soll sichergestellt werden, dass nicht beliebige Aussagen als wissenschaftliche Erkenntnisse über die Untersuchungsgegenstände akzeptiert werden, insbesondere sollen triviale Tautologien vermieden werden, welche wahr, aber nicht gehaltvoll sind. Wahr heißen Aussagen dann, wenn ihr Gehalt auch tatsächlich zutrifft. Damit soll sichergestellt werden, dass 
keine gehaltvollen, aber falschen Aussagen als wissenschaftliche Erkenntnisse akzeptiert werden. ${ }^{1}$ Das Kriterium der Wahrheit einer Aussage kann in diesem Zusammenhang sehr weit gefasst werden. Es ist nicht nötig, sich auf dieser Ebene auf eine bestimmte Wahrheitskonzeption festzulegen, abhängig vom Gegenstandsbereich oder der sprachlichen Konventionen der Fachdisziplin können auch Validität, Richtigkeit, Plausibilität oder Korrektheit als Kriterien verwendet werden. Unabhängig von der verwendeten Terminologie ist jedoch der Umstand, dass Wahrheitsansprüche einer Begründung oder Rechtfertigung bedürfen.

Wissenschaftliche Erkenntnisse umfassen den so abgesteckten Bereich der gehaltvollen Wahrheit. Hypothesen sind gehaltvolle Aussagen, die im Forschungsprozess als Kandidaten für wissenschaftliche Erkenntnis aufgestellt werden. Der wissenschaftliche Prozess hat nun die Aufgabe, Hypothesen aufzustellen und zu überprüfen.

Diese formale Bestimmung des wissenschaftlichen Prozesses macht keinerlei Aussagen darüber, wie in der Forschungspraxis Hypothesen aufgestellt und überprüft werden könne oder sollen. Es soll auch nicht suggeriert werden, dass es eine einzige wissenschaftliche Methode gebe. ${ }^{2}$ Vielmehr soll die Diskussion wissenschaftstheoretischer Positionen dazu führen, angesichts der faktischen Methodenpluralität in den Wissenschaften, die Elemente und Zusammenhänge der wissenschaftlichen Arbeitsabläufe besser zu verstehen und gegebenenfalls durch weitere Methodenreflexion im Einzelfall zu korrigieren.

Doch wie lässt sich der konkrete Forschungsprozess adäquat fassen? William Whewell macht den Vorschlag, den Prozess und das Produkt der Forschung als wissenschaftliche Entdeckung, scientific discovery, folgendermaßen in drei Phasen aufzuteilen (Whewell 1840):

1. Am Anfang steht ein glücklicher Einfall.

2. Dieser Einfall wird artikuliert und zu einer Hypothese ausformuliert.

3. Die Hypothese wird im Fall ihrer erfolgreichen Überprüfung als Erkenntnis anerkannt.

1 Zur selben Problemklasse gehören auch überangepasste Modelle, die sehr gute Ergebnisse auf den Trainingsdaten, aber nur schlechte Ergebnisse auf den Testdaten erzielen. Das Modell macht gehaltvolle Aussagen über die Testdaten, die jedoch nicht zutreffen.

2 Die Behauptung, dass die Wissenschaft durch eine einzige, universelle Methode bestimmt wird, hat schon Karl Popper zu deutlichen Worten inspiriert. So soll er am ersten Vorlesungstag in den Hörsaal gekommen sein und gerufen haben: "I am a professor of scientific method, and I have a problem. There is no such thing as scientific method!” (Nickles 2006, S. 177) 
Der glückliche Einfall (1) oder happy thought, wie ihn Whewell nennt, zeichnet sich durch das Fehlen von Regeln aus:

Scientific Discovery must ever depend upon some happy thought, of which we cannot trace the origin; some fortunate cast of intellect, rising above all rules. No maxims can be given which inevitably lead to discovery. (Whewell 1840, S. 186)

Es kann ihm zufolge nicht eine einzige Maxime oder Methode geben, die unweigerlich zu einer Entdeckung führt, es gibt keine Anleitung, um Heureka-Momente zu erzwingen. Die happy thoughts sind nicht determiniert, allerdings sind sie auch nicht beliebig, wie Schickore (2018, Abs. 3) bemerkt:

In this sense, happy thoughts are accidental. But in an important sense, scientific discoveries are not accidental. The happy thought is not a wild guess. Only the person whose mind is prepared to see things will actually notice them. The "previous condition of the intellect, and not the single fact, is really the main and peculiar cause of the success. The fact is merely the occasion by which the engine of discovery is brought into play sooner or later. It is, as I have elsewhere said, only the spark which discharges a gun already loaded and pointed; and there is little propriety in speaking of such an accident as the cause why the bullet hits its mark.” (Whewell 1840, S. 189)

Die engine of discovery sollte nicht als Mechanismus verstanden werden, der automatisch Erkenntnisse generiert, sondern als Hintergrundtheorien, Domänenwissen und bekannte Methoden, die - auch in den digitalen Geisteswissenschaften den Kontext konstituieren, der Einfälle überhaupt möglich macht. Hier kann die CRETA-Werkstatt (vgl. Einleitung dieses Bandes) als Beispiel dienen, die Einfälle und Heureka-Momente haben sich oft bei Thementischen und brainstormingSitzungen eingestellt, wenn Domänenexpert:innen aus den verschiedenen Fachdisziplinen über gemeinsame Themen nachdenken und dabei Hintergrundwissen, Theorien und Methoden miteinander verbinden. Durch solches Vorgehen kann kein Ergebnis (und erst recht kein Ergebnisinhalt) vorausbestimmt werden, jedoch kann der Arbeitsprozess so strukturiert werden, dass die Wahrscheinlichkeit eines Einfalls steigt. Der happy thought (1) ist keine mysteriöse Eingebung, die dem wissenschaftlichen Genie zufällt, sondern Teil der Forschungspraxis.

Der Übergang vom happy thought zur Erkenntnis (2) ist der für den Arbeitsablauf wichtigste und gleichzeitig am wenigsten bestimmte Schritt. Hier stellt sich die Frage, ob er rational analysierbar ist oder ausschließlich durch erkenntnisexterne Faktoren bestimmt wird. Der Arbeitsablauf der reflektierten Textanalyse ist, wie Kuhn (2020) und Pichler und Reiter (2020) in diesem Band ausgeführt haben, hinreichend systematisch, um eine Aufteilung in Module und Handlungsanweisungen zu ermöglichen. 
Die Rechtfertigung in (3), wo die Hypothesen systematisch evaluiert werden, findet in der reflektierten Textanalyse jedoch an verschiedenen Stellen statt, zum einen zeitlich getrennt am Ende des prototypischen Arbeitsablaufes, wenn die Befunde interpretiert werden zum anderen logisch in den jeweiligen Teilmodulen, die entweder ihre eigenen Validierungsverfahren mitbringen oder selbst durch Rückführung auf die Standards der Fachdisziplinen gerechtfertigt sind.

Die Abbildung der reflektierten Textanalyse auf das 3-Phasen-Modell Whewells zeigt auch dessen Schwächen: Zum einen folgt der tatsächliche Arbeitsablauf nicht streng den drei Phasen, zum anderen ist nicht klar, wie genau zwischen Prozess oder Ergebnis differenziert werden kann. Gehört die Rechtfertigung zur Entdeckung oder ist sie unabhängig?

\section{Entdeckungs- und Rechtfertigungszusammenhang}

Eine Antwort auf die Frage, wie Rechtfertigung und Entdeckung zusammenhängen bietet Hans Reichenbach an, der in Experience and Prediction (1938) die begriffliche Unterscheidung zwischen context of discovery (Entdeckungs- oder Entstehungszusammenhang) und context of justification (Rechtfertigungs- oder Begründungszusammenhang) einführt, um die Geltung wissenschaftlicher Erkenntnisse von ihren sozialen, ökonomischen und psychologischen (und anderen erkenntnisexternen) Entstehungsumständen zu trennen.

Der Entdeckungszusammenhang umfasst die tatsächlichen Denkvorgänge und Prozesse, die zu einer Entdeckung geführt haben, und ist Gegenstand der Psychologie, Soziologie und Geschichtswissenschaft, nur der Rechtfertigungszusammenhang ist nach Reichenbach genuiner Gegenstand der Wissenschaftstheorie, er umfasst die gesamte Bewertung und Überprüfung der wissenschaftlichen Erkenntnisse. Dieser Unterscheidung liegt die Vorannahme zugrunde, dass die Wissenschaftstheorie ein normatives Projekt sei, das formallogisch verfährt und dessen Gegenstände die rationalen Rekonstruktionen wissenschaftlicher Theorien sind. Rationale Rekonstruktion bedeutet, dass die Bewertung der Gültigkeit und Zuverlässigkeit einer Theorie anhand einer fiktionalen Konstruktion durchgeführt wird, bei der die tatsächlichen Denkvorgänge und sprachlichen Äußerungen durch logische Konstruktionen ersetzt werden, da selbst die vollständig ausformulierten wissenschaftlichen Ergebnisse in Veröffentlichungen noch zu unpräzise sind oder noch subjektive Motive enthalten (Reichenbach 1938, S. 6). Hierzu bemerkt Reichenbach: 
If a more convenient determination of this concept of rational reconstruction is wanted, we might say that it corresponds to the form in which thinking processes are communicated to other persons instead of the form in which they are subjectively performed. [The] wellknown difference between the thinker's way of finding [a] theorem and his way of presenting it before a public may illustrate the difference in question. I shall introduce the terms context of discovery and context of justification to mark this distinction. Then we have to say that epistemology is only occupied in constructing the context of justification. But even the way of presenting scientific theories is only an approximation to what we mean by the context of justification. Even in the written form scientific expositions do not always correspond to the exigencies of logic or suppress the traces of subjective motivation from which they started. (Reichenbach 1938, S. 6-7)

Das Problem, das durch die Unterscheidung von Entdeckungs- und Rechtfertigungszusammenhang primär gelöst werden soll, zeigt sich am Beispiel August Kekulés deutlich, der die Struktur des Benzolrings im Traum erkannt haben will:

Ich drehte den Stuhl nach dem Kamin und versank in Halbschlaf. Wieder gaukelten die Atome vor meinen Augen. Kleinere Gruppen hielten sich diesmal bescheiden im Hintergrund. Mein geistiges Auge, durch wiederholte Gesichte ähnlicher Art geschärft, unterschied jetzt grössere Gebilde von mannigfacher Gestaltung. Lange Reihen, vielfach dichter zusammengefügt; Alles in Bewegung, schlangenartig sich windend und drehend. Und siehe, was war das? Eine der Schlangen erfasste den eigenen Schwanz und höhnisch wirbelte das Gebilde vor meinen Augen. Wie durch einen Blitzstrahl erwachte ich; auch diesmal verbrachte ich den Rest der Nacht um die Consequenzen der Hypothese auszuarbeiten. (August Kekulé nach Schultz 1890, S. 1306)

Auch wenn die Entdeckungen in den digitalen Geisteswissenschaften selten im Traum gemacht werden, kommt die Unterscheidung zwischen Entdeckung und Rechtfertigung auch hier zum Tragen: Die Ergebnisse komputationeller Werkzeuge können nicht für sich allein stehen, sondern müssen in eine Argumentation eingebunden sein und interpretiert werden. Dieser Aspekt der Rechtfertigung findet sich im Schema der reflektierten Textanalyse als „Interpretation der Befunde“ (Pichler und Reiter 2020, S. 54). Da die Interpretation der Befunde Teil des Arbeitsablaufes ist und nicht nach der Formulierung der Endergebnisse einsetzt, ist es nötig, genauer auf das Verhältnis zwischen Entdeckungs- und Rechtfertigungszusammenhang einzugehen.

In der „orthodoxen“ (Feigl 1970) Lesart, ${ }^{3}$ welche die wissenschaftstheoretischen Diskussionen lange geprägt hat, werden Entdeckung und Rechtfertigung als zeitlich und logisch streng getrennt aufgefasst: Etwas muss zuerst entdeckt werden, bevor es gerechtfertigt werden kann. Dabei spielen Logik und normati-

3 Wie Reichenbach selbst die Kontextunterscheidung verstanden hat, ist Gegenstand einer eigenen Debatte, vgl. Nickles 1980 und Schiemann (2006). 
ve Analysen ausschließlich im Rechtfertigungszusammenhang eine Rolle. Diese Lesart ist mit vier Schwierigkeiten konfrontiert:

1. Wissenschaftliche Prozesse laufen in der Regel nicht in genau zwei Schritten ab: Häufig alterieren Teilentdeckungen und Teilrechtfertigungen (HoyningenHuene 2006, S. 120-121).

2. Die beiden Phasen lassen sich nicht immer trennscharf unterscheiden: Neue Messmethoden, die die Verbesserung eines empirischen Gesetztes zur Folge haben, können sowohl als Entdeckung betrachtet werden, als auch als Beitrag zur Rechtfertigung des Gesetzes (Hoyningen-Huene 2006, S. 120-121; Arabatzis 2006, S. 216).

3. Entdeckungen werden zumeist nicht zufällig gemacht, sondern folgen logischen und methodologischen Überlegungen und Verfahren (Nickles 1980, S. 14).

4. Der Begriff der Entdeckung selbst ist schon normativ aufgeladen, denn nicht jede Behauptung ist eine Entdeckung, sie wird es erst, wenn sie durch die Forschungsgemeinschaft als solche anerkannt wird (Arabatzis 2006, S. 217).

Die statische Auffassung, dass die Entdeckung zeitlich vor der Rechtfertigung einsetzt, muss so erweitert werden, dass komplexere Arbeitsweisen berücksichtigt werden können. Für die reflektierte Textanalyse bedeutet das, dass Entdeckung und Rechtfertigung auf Modulebene relativ zu den beteiligten Fachdisziplinen angesetzt werden. Jedes Modul, d. h. jeder Arbeitsschritt, der ein Ergebnis produziert, muss selbst gerechtfertigt sein, um zu gewährleisten, dass das Endergebnis wahre, gehaltvolle Aussagen über den Untersuchungsgegenstand enthält.

In den nächsten beiden Abschnitten werden nun die systematischen Aspekte von Entdeckung (Abschnitt 4) und Rechtfertigung (Abschnitt 5) weiter ausdifferenziert, um die Arbeitsabläufe der reflektierten Textanalyse adäquat abbilden zu können.

\section{Entdeckungszusammenhang}

Die Unterscheidung zwischen Entdeckungs- und Rechtfertigungszusammenhang findet eine Entsprechung in der Formulierung der hypothetisch-deduktiven (H-D) 
Methode, wobei die Formulierung von Hypothesen ${ }^{4}(\mathrm{H})$ dem Entdeckungszusammenhang und die deduktive Überprüfung (D) dem Rechtfertigungszusammenhang zugeordnet werden kann. Bei der deduktive Überprüfung der Hypothesen werden logische Konsequenzen deduktiv abgeleitet und mit Beobachtungen verglichen. Dieses Verfahren findet sich zum Beispiel bei der Evaluation formaler Modelle, etwa bei der Bestimmung von precision- und recall-Metriken.

In der wissenschaftstheoretischen Untersuchung wird zumeist die Hypothesenbildung ausgeklammert, da es für die deduktive Überprüfung irrelevant ist, wie die zu prüfende Hypothese gefunden wurde. Bei der Analyse wissenschaftlicher Arbeitsabläufe ist die Art und Weise, wie Hypothesen aufgestellt werden, jedoch hochgradig relevant.

Einen Schritt hin zu einer Logik der Entdeckung geht Norwood Hansons in The Logic of Discovery:

H-D [hypothetico-deductive] accounts all agree that physical laws explain data, but they obscure the initial connexion between data and laws; indeed, they suggest that the fundamental inference is from higher-order hypotheses to observation statements. This may be a way of setting out one's reasons for accepting an hypothesis after it is got, or for making a prediction, but it is not a way of setting out reasons for proposing or for trying an hypothesis in the first place. Yet the initial suggestion of an hypothesis is very often a reasonable affair. It is not so often affected by intuition, insight, hunches, or other imponderables as biographers or scientists suggest. Disciples of the H-D account often dismiss the dawning of an hypothesis as being of psychological interest only, or else claim it to be the province solely of genius and not of logic. They are wrong. If establishing an hypothesis through its predictions has a logic, so has the conceiving of an hypothesis. (Hanson 1958, S. 71)

Das vorrangige Interesse an einer Entdeckungslogik besteht darin, den Entdeckungsprozess durch reliable Arbeitsabläufe so $\mathrm{zu}$ gestalten, dass möglichst richtige Hypothesen generiert werden. Doch auch wenn die Möglichkeit einer Entdeckungslogik eingeräumt wird, könnte bestritten werden, dass sie relevant für den Erkenntnisprozess ist, da die aufgestellten Hypothesen zusätzlich noch gerechtfertigt werden müssen, gleichgültig durch welche Prozedur sie generiert wurden.

Dieser Einwand kann jedoch entkräftet werden (Kelly 1987): Eine Prämisse des Einwands besagt, dass die Prozeduren, die Hypothesen aufstellen, und Prozeduren, die Hypothesen testen, unabhängig sind. Darum ist es notwendig, jede Hypothese zu testen, egal wie sie zustande gekommen ist. Gegeneinwand: Man kann zeigen, dass Überprüfung und Erzeugung der Hypothesen symmetrisch

4 Hypothese soll sehr weit als Kandidat für eine Erkenntnis verstanden werden, d. h. als Aussage, deren Richtigkeit unterstellt, aber noch nicht gezeigt ist. 
sind, denn jede Testprozedur kann in einen Generator transformiert werden. Kelly (1987, S. 440) zeigt das an einem trivialen Beispiel: Gegeben ist eine Relation $\varphi(x, y)$, die $y=x^{2}$ berechnet. Der Hypothesengenerator könnte einfach für jedes $x$ die Reihe der natürlichen Zahlen durchlaufen, während die Testprozedur die falschen Werte aussondern und die richtigen Werte behalten würde. Mit anderen Worten, die Testprozedur berechnet intern $y=x^{2}$, d. h. sie benutzt einen effizienten Generator als Subroutine. Dieses Beispiel zeigt, dass die strikte Unterscheidung nicht prinzipiell aufrechterhalten werden kann.

Ein noch allgemeineres Argument für die Möglichkeit einer Entdeckungslogik entwickelt Jantzen (2015): Angenommen, eine Entdeckungslogik wäre unmöglich, dann wäre der Prozess der Hypothesenbildung äquivalent mit einem vollständig zufälligen Prozess. Wird eine Hypothese zufällig aus der unendlichen Menge aller möglichen Hypothesen ausgewählt, dann tendiert die relative Wahrscheinlichkeit, die richtige Hypothese zu ziehen, gegen null. Die Wissenschaftsgeschichte ist jedoch voll von erfolgreichen Beispielen, in denen nach nur sehr wenigen Versuchen die richtige Hypothese gefunden wurde. Nur ein Wunder könnte die Tatsache erklären, dass die Wissenschaften empirisch so erfolgreich sind. Wunder sind als Erklärungsgründe ausgeschlossen. Daraus folgt, dass es eine Entdeckungslogik geben muss.

Diese beiden Argumente betonen die Relevanz der logischen Untersuchung des Entdeckungszusammenhangs hinsichtlich effizienter Methoden, Hypothesen zu generieren. Dennoch sollte die Möglichkeit einer Entdeckungslogik nicht so missverstanden werden, dass sie die Möglichkeit einer deterministischen Entdeckungsmaschine (Curd 1980, S. 207) implizieren würde. Also einer Maschine, die unweigerlich wahre und gehaltvolle wissenschaftliche Hypothesen generieren könnte. Stattdessen soll lediglich gezeigt werden, dass es Prozeduren, Algorithmen und Heuristiken der Theoriekonstruktion und Hypothesenbildung gibt, die systematisch untersucht werden können und so zu einer Methodenreflexion beitragen.

Doch was soll unter Entdeckungslogik verstanden werden? Die klassische deduktive Logik kommt offensichtlich nicht infrage, da deduktive Kalküle ausschließlich wahrheitserhaltend von den Prämissen unter Zuhilfenahme von Schlussregeln zu den Konklusionen übergehen, d.h. in den Konklusionen findet sich nichts, was nicht schon in den Prämissen gewesen wäre. Es wird nichts Neues hinzugefügt. Entdeckungen sind jedoch per Definition neu und zuvor unbekannt. Um Entdeckungen zu modellieren, werden also logische Schlussmuster benötigt, die den Gehalt der Prämissen inferenziell erweitern können.

Solche Schlussmuster finden sich bei Charles Sanders Peirce, der (1) Deduktion, (2) Induktion und (3) Abduktion unterscheidet. Peirce erläutert die drei Schlussmuster anhand des folgenden Beispiels (Peirce 1931-1958, CP 2.623): In 
einem Raum befinden sich auf einem Tisch eine Handvoll weißer Bohnen und in einer Ecke ein Beutel mit ausschließlich weißen Bohnen. Mithilfe der Bohnen $(x)$ und der Prädikate „stammt aus dem Beutel“ $(B)$ und „ist weiß“ $(W)$ können nun alle drei Schlussmuster gebildet werden.

1. Im Fall der Deduktion ist bekannt, dass alle Bohnen im Beutel weiß sind und dass die Bohnen auf dem Tisch aus dem Beutel stammen. Es kann mit Sicherheit geschlossen werden: Diese Bohnen sind weiß. (Der Schluss ist gewiss, wahrheitserhaltend und nicht erkenntniserweiternd.)

\begin{tabular}{rll} 
1. & $\forall x(B x \rightarrow W x)$ & Regel \\
2. & $B x$ & Fall \\
\hline$\therefore$ & $W x$ & Resultat
\end{tabular}

2. Im Fall der Induktion ist bekannt, dass die Bohnen aus dem Beutel stammen und dass sie weiß sind. Es kann die Regel erschlossen werden, dass alle Bohnen im Beutel weiß sind. (Der Schluss ist erkenntniserweiternd, ungewiss und nicht wahrheitserhaltend.)

$$
\begin{array}{rll}
\text { 1. } & B x & \text { Fall } \\
2 . & W x & \text { Resultat } \\
\hline \therefore & \forall x(B x \rightarrow W x) & \text { Regel }
\end{array}
$$

3. Im Fall der Abduktion ist bekannt, dass alle Bohnen im Beutel weiß sind und dass die Bohnen auf dem Tisch weiss sind. Es kann auf die Hypothese geschlossen werden: Die Bohnen stammen aus dem Beutel. (Der Schluss ist erkenntniserweiternd, ungewiss und nicht wahrheitserhaltend.)

$$
\begin{array}{lll}
\text { 1. } & \forall x(B x \rightarrow W x) & \text { Regel } \\
2 . & W x & \text { Resultat } \\
\hline \therefore & B x & \text { Fall }
\end{array}
$$

Oft werden die beiden erkenntniserweiternden, unsicheren und nicht wahrheitserhaltenden Schlussmuster, Induktion und Abduktion, zusammengefasst, wobei die Abduktion als Sonderfall der Induktion gesehen wird. Es gibt jedoch einen wichtigen Unterschied zwischen beiden Schlussmustern hinsichtlich des Ziels. Die Induktion zielt auf zukünftig beobachtbare Ereignisse ab, während die Abduktion unbeobachtete Ursachen oder explanatorische Gründe zum Ziel hat (Schurz 2008, S. 202).

Mit Magnani (2001, S. 20) können zwei Typen der Abduktion unterschieden werden: Selektive und kreative Abduktion. Die selektive Abduktion wählt aus 
einer gegebenen Menge von Hypothesen die beste Erklärung aus, während die kreative Abduktion neue Begriffe, Hypothesen oder Modelle erzeugt. Das Beispiel der Krankheitsdiagnose verdeutlicht den Unterschied: Die selektive Abduktion geht von den beobachteten Symptomen zur Diagnose, indem aus einer Liste mit Krankheitsbildern dasjenige ausgewählt wird, das den Symptomen am besten entspricht. Kreative Abduktion erzeugt hingegen eine neue Krankheitsdefinition aus dem beobachteten Krankheitsbild. Selektive Abduktion liefert immer minimal plausible Hypothesen, die dann durch weitere deduktiven Tests überprüft werden können.

In diesem Sinne kann selektive Abduktion als Schluss auf die beste Erklärung (SBE) verstanden werden, die sich jedoch von der ursprünglichen Konzeption Harmans (1965) hinsichtlich ihrer entdeckenden Funktion unterscheidet, denn Harmans SBE ist nur dann erfolgreich, wenn alle möglichen Alternativen zur Verfügung stehen. Da das jedoch nicht der Fall ist, kann nur auf die beste verfügbare Erklärung geschlossen werden, dieser Schluss auf die beste verfügbare Erklärung (SBVE) unterliegt folgender Einschränkung: Es ist nicht gewährleistet, dass die beste verfügbare Erklärung auch eine gute, d. h. zutreffende, Erklärung ist. Gerade bei neuen oder schlecht verstandenen Phänomenen ist die beste Erklärung oft reine Spekulation. ${ }^{5}$

Eine Lösung dieses Problems besteht darin, die entdeckende Rolle der Abduktion genauer zu betrachten. Jedes Schlussmuster hat eine rechtfertigende und eine entdeckende Funktion. Die rechtfertigende Funktion der Deduktion ist maximal, während ihre rechtfertigende Funktion minimal ist, d.h., sie liefert keine neuen Erkenntnisse, sondern nur maximale Gewissheit über die Wahrheit der Konklusion, da diese nichts enthält, was nicht schon in den Prämissen gewesen wäre. Bei der Abduktion verhält es sich genau umgekehrt, die Gewissheit der von ihr generierten Hypothesen ist minimal ${ }^{6}$, während ihre entdeckende Funktion maximal ist. In diesem Zusammenhang wäre es jedoch falsch, die minimale Rechtfertigungsfunktion der Abduktion so zu verstehen, dass sie gar keine begründende Kraft hätte, denn in der Praxis werden meistens nach erstaunlich wenigen Versuchen befriedigende Lösungen für ein Problem gefunden, was nahelegt, dass die abduktiven Suchprozesse sogar (relativ) effizient sind. Versuchte man, alle möglichen Erklärungen komputationell zu finden, würde der Suchraum exponentiell anwachsen, darum ist es notwendig, die Suche so einzuschränken, dass Lösun-

5 Schurz (2008, S. 203) führt hier intentionales Handeln übernatürlicher Wesen zur Erklärung von Naturphänomenen als Beispiel an.

6 Peirce (1903, CP 5.171) hält die abduktiv gewonnenen Hypothesen nicht einmal für wahrscheinlich, sondern lediglich für möglich. 
gen in kurzer Zeit gefunden werden können. ${ }^{7}$ Die effiziente Einschränkung des Suchraums kann nun wiederum als schwache Rechtfertigung für die gewonnenen Ergebnisse gelten (vgl. Schurz 2008, S. 204).

Die logischen Schlussmuster Deduktion, Induktion sowie selektive und kreative Abduktion erlauben eine differenzierte Beurteilung der Module und Teilschritte der reflektierten Textanalyse und können sowohl im Design also auch in der Umsetzung von konkreten Forschungsprojekten eingesetzt werden, um die inferenzielle Stringenz des Arbeitsablaufes zu bewerten. ${ }^{8}$

\section{Rechtfertigungszusammenhang}

Der Rechtfertigungszusammenhang enthält bei Reichenbach nur die epistemische Validierung der im Entdeckungszusammenhang gebildeten Hypothesen. Doch bei konkreten Arbeitsabläufen zeigt sich, dass nicht alle normativen Bewertungen epistemisch sind.

Im Forschungsprozess sind ständig Entscheidungen zu treffen und Evaluationen vorzunehmen. Doch nicht alle diese Evaluationen und Entscheidungen haben das Ziel, die Wahrheit einer Hypothese zu stützen. Eine nützliche Differenzierung des Rechtfertigungszusammenhangs nimmt Nickles (2006) vor, der darauf hinweist, dass nicht alle Evaluationen epistemische Rechtfertigungen sind. Er schlägt vor, zwei Bewertungstypen zu unterscheiden: (1) epistemische und (2) heuristische Bewertungen.

Epistemische Bewertungen betreffen alle Fragen nach der Wahrheit von Behauptungen. Heuristische Bewertungen hingegen sind Evaluationen und Entscheidungen, die aus pragmatischen oder forschungsökonomischen Gründen getroffen werden. Die meisten Forschungsentscheidungen werden nicht allein aus epistemischen Gründen getroffen, da es immer auch nötig ist, die Fruchtbarkeit und Durchführbarkeit der Forschungsansätze zu bedenken.

Bei der Begutachtung von Forschungsprojekten oder Antragsstellung steht die Wahrheit einer Hypothese selten im Vordergrund der Überlegungen (auch oder gerade, weil die Forschungsergebnisse noch nicht vorliegen). Vielmehr wird die Durchführbarkeit des Forschungsvorhabens, die Qualität des Forschungsdesigns oder die Wahrscheinlichkeit, dass der principle investigator das Projekt zu

7 Schurz (2008, S. 211) schlägt hier technische Lösungen wie Bestensuchen und Tableaukalküle als aussichtsreiche Kandidaten vor.

8 Zur Rolle der Abduktion als Heuristik in DH-Projekten siehe Gius und Jacke 2015 und Gius 2019. 
Ende bringen kann, beachtet. Es wird gefragt: Wie interessant oder wichtig ist die Fragestellung? Ist sie anschlussfähig? Sind Folgeprojekte möglich?

Heuristische Bewertungen beinhalten Überlegungen, die von epistemischen Beurteilungen entweder unabhängig sind oder vom Standpunkt zukünftiger Fruchtbarkeit her urteilen. Einstein verwendete zum Beispiel in seinem Aufsatz Über einen die Erzeugung und Verwandlung des Lichtes betreffenden heuristischen Gesichtspunkt (1905) die Formel für die ,schwarze Strahlung، von Wilhelm Wien, obwohl sie schon damals als nicht gültig verworfen war. Allerdings war sie für Einstein höchst fruchtbar, denn er konnte sie benutzen, um seine eigene Argumentation voranzubringen (Nickles 2006, S. 162). Und umgekehrt verlassen Wissenschaftler ihre Forschungsgebiete, obwohl sich die dort erlangten Erkenntnisse nicht als falsch erwiesen haben und obwohl es noch offene Probleme gibt, weil sie größere zukünftige Erfolge in anderen Gebieten erwarten. Max Delbrück kehrte beispielsweise der Physik den Rücken zu und betätigte sich in der Biologie, nicht etwa, weil die Physik fehlgeschlagen wäre oder es keine offenen Probleme mehr gab, sondern weil er die Möglichkeit, selbst fruchtbar zu arbeiten, dort ungünstiger einschätzte als in der Biologie (Nickles 2006, S. 162).

In der Planung und Organisation spielt die Wahl des nächsten Forschungsvorhabens eine kritische Rolle. Welches der vielen offenen Probleme, welche der vielen interessanten Fragen soll als nächstes bearbeitet werden? Hier hilft die rein epistemische Maxime ,Finde die Wahrheit!' nicht weiter. Hier helfen nur heuristische Überlegungen (Nickles 2006, S. 163).

Gerade in multidisziplinären Kooperationen, die die digitalen Geisteswissenschaften prägen, ist der Bereich der Heuristik von großer Bedeutung, unter anderem bei der Wahl der Korpora und der komputationellen Werkzeuge. ${ }^{9}$

\section{Anwendung}

Der im CRETA-Projekt entwickelte prototypische Arbeitsablauf versteht sich vorrangig als methodisch-arbeitspraktische Herangehensweise, um die Probleme mehrdimensionaler, interdisziplinärer Forschungsvorhaben zu bewältigen. Sie beansprucht dabei nicht, vollendete Theorie oder letztgültige Methodologie zu sein, sondern versucht vielmehr, anhand eines differenzierten, prototypischen Arbeitsablaufes eine reflektierte wissenschaftliche Praxis $\mathrm{zu}$ entwickeln. Dementsprechend sollen die oben angestellten wissenschaftstheoretischen Überlegungen nicht spezifische Ergebnisse oder Entdeckungen thematisieren, sondern

9 Zur Rolle von Heuristiken in den Digital Humanities vgl. Gius und Jacke 2015. 
einen begriffliche Rahmen zur Verfügung stellen, um interdisziplinäre Abstimmungsprozesse zu erleichtern.

Eine Besonderheit des CRETA-workflows ist die Modularisierung des Arbeitsablaufs, welche als Zerlegung in eindeutige Handlungsvorschriften konzipiert ist (Pichler und Reiter 2020, S. 43). Der Forschungsprozess wird also normativ strukturiert, um die Ziele der jeweiligen konkreten DH-Projektarbeit zu erreichen. Es geht aus wissenschaftstheoretischer Perspektive darum, die einzelnen Module so zu verzahnen, dass eine lückenlose Rechtfertigungskette hergestellt wird, die von den neuen Entdeckungen zurück zu bereits akzeptierten Erkenntnissen in den beteiligten Fachdisziplinen reicht.

So zeigt zum Beispiel Trevor Owens (2012) anhand einer Diskussion über topic modeling, dass die Bedingungen, unter denen ein technisches Instrument Daten produziert hat, notwendige Voraussetzungen sind, um beurteilen zu können, inwieweit jene Daten als Mittel zur Rechtfertigung einer These oder der Verteidigung einer Interpretation dienen können. Er bemerkt in diesem Zusammenhang:

When we separate out the context of discovery and exploration from the context of justification we end up clarifying the terms of our conversation. There is a huge difference between "here is an interesting way of thinking about this" and "This evidence supports this claim." Both scientists and humanists make both of these kinds of assertions. (Owens 2012)

Die Unterscheidung zwischen Entdeckung und Rechtfertigung kann dabei helfen, zu klären, was ein jeder Einzelschritt zum gesamten Forschungsprozess beiträgt. Liefert er etwas Neues? Handelt es sich um eine Entdeckung? Oder hilft er dabei, eine gemachte Entdeckung abzusichern? Liefert er Gründe für die Gültigkeit einer bestimmten Aussage?

Die oben gewonnenen Begriffe können nun für die Methodenreflexion im CRETA-Projekt angewendet werden. Der prototypische Arbeitsablauf der reflektierten Textanalyse kann auf das obige Modell wie folgt abgebildet werden:

1. Die Aufteilung der disziplinären Fragestellung in Teilfragen und Module wird von heuristischen Bewertungen geleitet: In erster Linie geht es darum, herauszufinden, welche Probleme mit den verfügbaren Ressourcen bearbeitet werden können und welche Fragestellungen fruchtbar erscheinen. Ausschlaggebend ist unter anderem, welche Mittel zur Verfügung stehen (sowohl Techniken als auch Mitarbeiter), welche Themen gerade in der wissenschaftlichen Gemeinschaft diskutiert werden und welche Interessen die Kooperationspartner haben. Ein großes Projekt mit vielen Hilfskräften, die Annotationen machen können, wird andere Fragen auswählen als ein kleines Projekt mit einem neuen Visualisierungstool. 
2. Die Operationalisierung der Begriffe erfolgt zunächst abduktiv: Ein Begriff ist denn operationalisiert, wenn eine Menge an Indikatoren (z. B. Textoberflächeneigenschaften) und Regeln gefunden wurde, die mit hinreichender Wahrscheinlichkeit auf die korrekte Verwendung des Begriffs hinweisen. Immer dann, wenn die Indikatoren und ihre regelhaften Verknüpfungen am Text aufgewiesen werden können, ist auch die Verwendung des Begriffs adäquat.

3. Ein Kernstück des prototypischen Arbeitsablaufes ist die Konstruktion der Korpora und die Erstellung der Annotationsrichtlinien: Die Erstellung der Annotationsrichtlinen kann als Schluss auf die beste Erklärung verstanden werden. Die Wahl der Texte und Annotationsrichtlinien folgt heuristischen Bewertungen, die auf den zukünftigen Erfolg der eingesetzten Mittel zielen. Die iterative Ausarbeitung und Stabilisierung der Annotationen erfolgt im Wechsel von abduktiver oder induktiver Regelaufstellung und deduktiver Prüfung der Regeln. Annotationen können als Inferenzen über den Text verstanden werden, die durch abduktive Schlüsse auf Basis der Annotationsrichtlinien erzeugt werden. Die Revision der Richtlinien selbst ist wiederum eine Instanz der selektiven Abduktion: Es wird aus den vorliegenden Annotationen diejenige ausgewählt, welche die beste Erklärung für die gegebenen Textphänomene liefern kann. Entscheidungsgründe der Annotator:innen und quantitative Metriken liefern anschließend die deduktive Rechtfertigung der jeweiligen Hypothese.

Jeder der Teilschritte ist entweder unabhängig gerechtfertigt oder als Schluss auf die beste Erklärung abgesichert. Die Aufteilung des Arbeitsablaufes in einzelne Komponenten kann somit garantieren, dass jeder Schritt, der einen weiteren rechtfertigt, selbst gerechtfertigt ist.

\section{Fazit}

Die digitalen Geisteswissenschaften haben sich vom romantischen Bild des solitären Genius weit entfernt. Die Arbeitsabläufe ähneln mehr denen der Naturwissenschaften, die in Laborsituationen arbeitsteilig verfahren, nur unter den verschärften Bedingungen der Interdisziplinarität. Die Verschärfung besteht in der Hauptsache darin, dass sich Erkenntnisziele, Methoden und Vokabulare oft signifikant in den Teildisziplinen unterscheiden. Um die methodische Verständigung zu erleichtern, wurde eine Reihe von Begriffsunterscheidungen entwickelt, die die 
Reflexion der eigenen Praxis unter dem Vorzeichen der Wissenschaftstheorie ermöglichen.

Mithilfe der Unterscheidung zwischen Entdeckungs- und Rechtfertigungszusammenhang, kreativer und selektiver Abduktion, Schluss auf die beste Erklärung sowie heuristischer und epistemischer Bewertung ist es möglich, praktische Fragen und Probleme auf einer theoretischen Ebene zu thematisieren.

\section{Literatur}

Arabatzis, Theodore (2006). „On the Inextricability of the Context of Discovery and the Context of Justification“. In: Revisiting discovery and Justification. Historical and philosophical perspectives on the context distinction. Hrsg. von Jutta Schickore und Friedrich Steinle. Dordrecht: Springer, S. 215-230.

Curd, Martin (1980). „The Logic of Discovery: An Analysis of Three Approaches“. In: Scientific Discovery, Logic, and Rationality. Hrsg. von Thomas Nickles. Dordrecht: Reidel, S. 201219.

Einstein, Albert (1905). „Über einen die Erzeugung und Verwandlung des Lichtes betreffenden heuristischen Gesichtspunkt“. In: Ann. Phys. 322.6, S. 132-148. DoI: 10.1002 / andp. 19053220607.

Feigl, Herbert (1970). „The ,orthodox“ view of theories: Remarks in defense as well as critique“. In: Analyses of Theories and Methods of Physics and Psychology. Hrsg. von Michael Radneri und Stephen Winokur. Bd. 4. Minnesota Studies in the Philosophy of Science. Minneapolis, MN: University of Minnesota Press, S. 3-16.

Gius, Evelyn (2019). „Computationelle Textanalysen als fünfdimensionales Problem: Ein Modell zur Beschreibung von Komplexität“. Pamphlet 8. Digital Humanities Cooperation. URL: https://www.digitalhumanitiescooperation.de/wp-content/uploads/2019/12/pamphlet_ gius_2.0.pdf (besucht am 1. Juni 2020).

Gius, Evelyn und Janina Jacke (2015). „Informatik und Hermeneutik. Zum Mehrwert interdisziplinärer Textanalyse“. In: Zeitschrift für digitale Geisteswissenschafteni 1: Sonderband der Zeitschrift für digitale Geisteswissenschaften. Hrsg. von Constanze Baum und Thomas Stäcker. Dol: 10.17175/sb001_006.

Hanson, Norwood Russell (1958). „The Logic of Discovery“. In: Journal of Philosophy 55.25, S. $1073-1089$.

Harman, Gilbert (1965). „The Inference to the Best Explanation“. In: The Philosophical Review 74.1, S. 88-95.

Hoyningen-Huene, Paul (2006). „Context of Discovery versus Context of Justification and Thomas Kuhn“. In: Revisiting discovery and Justification. Historical and philosophical perspectives on the context distinction. Hrsg. von Jutta Schickore und Friedrich Steinle. Dordrecht: Springer, S. 119-132.

Jantzen, Benjamin C. (2015). „Discovery without a ,logic' would be a miracle“. In: Synthese 193.10, S. 3209-3238.

Kelly, Kevin (1987). „The Logic of Discovery“. In: Philosophy of Science 54.3, S. 435-452. 
Kuhn, Jonas (2020). „Einleitung“. In: Reflektierte Algorithmische Textanalyse. Hrsg. von Nils Reiter, Axel Pichler und Jonas Kuhn. Berlin: De Gruyter, S. 9-40.

Magnani, Lorenzo (2001). Abduction, Reason and Science. Processes of Discovery and Explanation. New York: Kluwer.

Nickles, Thomas (1980). „Introductory Essay: Scientific Discovery and the Future of Philosophy of Science“. In: Scientific Discovery, Logic, and Rationality. Hrsg. von Thomas Nickles. Dordrecht: Reidel, S. 1-59.

Nickles, Thomas (2006). Heuristic Appraisal: Context of Discovery or Justification? Historical and philosophical perspectives on the context distinction. Hrsg. von Jutta Schickore und Friedrich Steinle. Dordrecht.

Owens, Trevor (2012). Discovery and Justification are Different: Notes on Science-ing the Humanities. URL: http: / / www.trevorowens .org / 2012 / 11/ discovery- and - justification - aredifferent-notes-on-sciencing-the-humanities/ (besucht am 1. Juni 2020).

Peirce, Charles Sanders (1903). „Lectures on Pragmatism“. In: Collected Papers. Hrsg. von Charles Hartshorne, Paul Weiss und Arthur Burks. 8 Bde. Cambridge, MA: Harvard University Press, S. 5.14-5.212.

Peirce, Charles Sanders (1931-1958). Collected Papers. Hrsg. von Charles Hartshorne, Paul Weiss und Arthur Burks. 8 Bde. Cambridge, MA: Harvard University Press.

Pichler, Axel und Nils Reiter (2020). „Reflektierte Textanalyse“. In: Reflektierte Algorithmische Textanalyse. Hrsg. von Nils Reiter, Axel Pichler und Jonas Kuhn. Berlin: De Gruyter, S. 4359.

Reichenbach, Hans (1938). Experience and Prediction. An Analysis of the Foundations and the Structure of Knowledge. Chicago: Chicago University Press.

Schickore, Jutta (2018). „Scientific Discovery“. In: The Stanford Encyclopedia of Philosophy. Hrsg. von Edward N. Zalta. Summer 2018. Metaphysics Research Lab, Stanford University. URL: https: / / plato . stanford . edu / archives / sum2018 / entries / scientific - discovery/ (besucht am 1. Juni 2020).

Schiemann, Gregor (2006). „Inductive Justification and Discovery. On Hans Reichenbach's Foundation of the Autonomy of the Philosophy of Science". In: Revisiting discovery and Justification. Historical and philosophical perspectives on the context distinction. Hrsg. von Jutta Schickore und Friedrich Steinle. Dordrecht: Springer, S. 23-40.

Schultz, Gustav (1890). „Feier der Deutschen Chemischen Gesellschaft zu Ehren August Kekulé’s“. In: Berichte der deutschen chemischen Gesellschaft 23.1, S. 1265-1312.

Schurz, Gerhard (2008). „Patterns of abduction“. In: Synthese 164.2, S. 201-234.

Whewell, William (1840). The Philosophy of the Inductive Sciences founded upon their history. Bd. 2. London: John Parker. 
